\title{
Immunolocalization of the calcium-sensing receptor in developing human kidney
}

\author{
Giovanna M. Crisi', Gary F. Rockwell ${ }^{2}$, Gregory L. Braden ${ }^{3}$ and Thomas J. Campfield ${ }^{2}$
}

BACKGROUND: The calcium-sensing receptor (CSR) is a G-protein receptor that plays a critical role in calcium regulation. In the kidney, the CSR regulates calcium reabsorption in the thick ascending limb, where stimulation of the CSR inhibits calcium reabsorption in response to increased calcium in the peritubular fluid. In the collecting duct, apical CSR activation may play a role in osmoregulation, increasing water excretion in response to increased luminal calcium.

METHODS: We studied the ontogeny of the CSR in developing human kidney using immunohistochemical methods.

RESULTS: The CSR is first expressed in the S-shaped body in the region destined to form the ascending limb and distal tubule. Other regions of the S-shaped body, as well as ureteric buds, do not express the CSR. The CSR is observed in thick ascending limb as early as 20 wk of development. The CSR is not observed in proximal tubule or collecting duct between 20 and 40 wk of human development.

CONCLUSION: During early human renal development, CSR expression is limited to the thick ascending $\operatorname{limb}$ and distal tubule, where this receptor may play a role in calcium homeostasis between 20 and 40 wk of human development.

$\mathbf{P}$ remature birth imposes stress on the kidney during a critical period of nephron formation and differentiation. The renal consequences of prematurity include impaired skeletal development due to abnormal renal calcium and phosphorus homeostasis (1); nephrocalcinosis; renal accumulation of calcium oxalate, which may lead to abnormal tubular function in childhood (2-10); and impaired nephron formation, leading to abnormal renal function in adulthood $(11,12)$.

The calcium-sensing receptor (CSR) is a G-proteincoupled receptor expressed mainly in parathyroid, calcitonin-secreting cells of the thyroid, bone, intestine, and kidney. The CSR detects changes in serum calcium and directly or indirectly helps normalize serum calcium level (13). Approximately $25 \%$ of calcium reabsorption occurs in the thick ascending limb (TAL) of the loop of Henle, where tubular fluid becomes electropositive relative to peritubular fluid, and calcium moves via paracellular pathways to the electronegative peritubular space $(14,15)$. The CSR, expressed along the basolateral portion of the TAL, senses increased peritubular calcium and inhibits calcium reabsorption via downregulation of the apical renal outer medullary potassium channel, dissipating the charge gradient required for calcium reabsorption, or via inhibition of vasopressininduced cAMP formation, which may affect permeability of the paracellular pathway (16). The critical importance of the CSR in renal calcium regulation is seen in patients with familial hypocalciuric hypercalcemia, in whom downregulating mutations of the CSR impair the ability of the CSR to respond to rising peritubular calcium levels, resulting in continued calcium absorption in the face of increased serum calcium (17).

It has been suggested that, in the mature collecting duct (CD), the CSR may play a role in preventing kidney stone formation by increasing water excretion in response to increased concentration of luminal calcium, leading to a decrease in urine calcium concentration (18). The CSR has been implicated in the pathogenesis of polyuria associated with hypercalcemia via this mechanism (19). However, the importance of the CSR in osmoregulation has been challenged. Lam et al. observed high urine osmolality and low urine volume in the presence of urine calcium concentrations greater than $5 \mathrm{mM} / \mathrm{l}(20)$, and Bergsland et al. found no difference in urine volume in idiopathic hypercalciuric stone-formers as compared with controls, even though the hypercalciuric stone-formers had a twofold greater urine calcium excretion (21). These observations suggest that urine calcium does not have a clinically significant role in osmoregulation. Diminished expression or responsiveness of the CSR in the CD could play a role in the pathogenesis of nephrocalcinosis, which has been reported to occur in up to $15 \%$ of premature infants (22).

Clinical disorders of calcium homeostasis occur in premature infants through mechanisms that may involve the CSR, but no data are available regarding expression of the CSR in developing human kidney. Therefore, we studied CSR localization in developing human kidney between 20 and $40 \mathrm{wk}$ of development using immunohistochemical methods. 


\section{RESULTS}

Sixteen autopsy cases in which death occurred between 20 and $40 \mathrm{wk}$ gestation that met inclusion criteria were identified. Of these, seven fetuses died as a result of intrauterine demise, and nine infants died as a result of complications of prematurity. Extrauterine survival was $\leq 1 \mathrm{~d}$ in 7 cases and $\leq 8 \mathrm{~d}$ in 2 cases. Gender distribution was 14 males and 2 females. Interval from death to fixation averaged $19 \mathrm{~h}$. The metanephric stage of kidney development was studied, which includes stage 3 (20-22 to $32-36$ wk gestation) and stage 4 (which continues into adult life). Mature non-neoplastic renal tissue was obtained from five tumor nephrectomies to evaluate potential changes in expression of CSR between developing and mature kidneys. The age range was 7-67 y (mean $41 \mathrm{y}$ ), including three females and two males. Unless otherwise specified, the results and figures represent immunolocalization of the CSR using the monoclonal antibody clone 5C10. Because individual structures showed either diffusely positive or diffusely negative immunostaining for the CSR antibodies (both monoclonal and polyclonal antibodies), a semiquantitative approach to evaluate staining was not used.

\section{CSR in Nephrogenic Zone}

Nephrogenic structures could be reliably evaluated up to $32 \mathrm{wk}$ gestational age in 13 cases; three cases represented 35 to $40 \mathrm{wk}$ gestational age, when the nephrogenic zone is not present. There was no immunostaining for the CSR in either Wolffian duct-derived ureteric buds, or in metanephric blastemaderived early structures, including vesicle, C-shaped body, and early S-shaped body (Figure 1a,b). Insets in Figure 1a,b show the lack of CSR staining in ureteric bud and vesicle, and positive PAX2 nuclear staining in these structures, respectively.
Immunoreactivity for the CSR was first identified in the upper limb of the S-shaped body during early elongation of the upper limb at the stage of hemispheric cavity formation between the lower and upper limbs (Figure 1b). At this stage of development, up to $14 \%$ of upper limbs showed weak cytoplasmic positivity for CSR. In more advanced stages of development, at the phase of Malpighian corpuscle formation, cytoplasmic CSR staining was present in approximately $80 \%$ of upper limbs. The cells of the upper limb differentiate into distinct distal tubule segments: macula densa (MD), distal convoluted tubule (DT), and ascending loops of Henle. CSR immunolocalization was observed in the same nephrogenic zone structures at 20 to $32 \mathrm{wk}$ gestational age.

\section{CSR in Developing and Mature Tubular Segments}

Proximal tubule (PT) segments, including convoluted (S1) and straight segments (S2 and S3), did not demonstrate any CSR staining at any stage of development. The middle limb of the S-shaped body, which develops into PT, was also negative for CSR staining (Figure $1 \mathbf{a}, \mathbf{b}$ ).

In developing loops of Henle, weak CSR staining was noted in cells just proximal to the bend of the loop of Henle, representing the boundary between the descending and ascending limb epithelium (Figure 1a). CSR was observed along the bend and in the TAL, MD, and DT. Within the medulla and renal papilla, large tubular structures representing CD were negative for CSR, whereas smaller loop of Henle tubules were positive for CSR and likely represent portions of the ascending limb in this stage of development (Figure 2a,b). Of note, the thin loop of Henle is distinguishable during fetal life only in the first few generations of nephrons and cannot be seen in older generations until after birth,

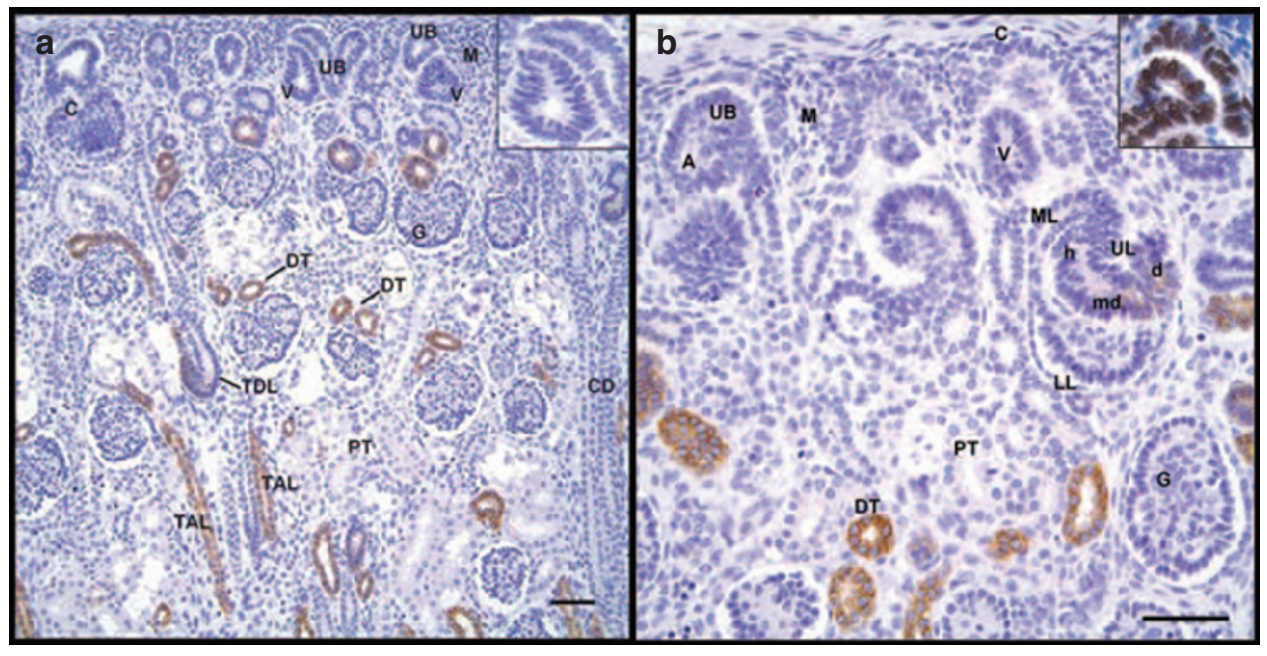

Figure 1. Calcium-sensing receptor (CSR) immunoreactivity in developing human kidney at 20 wk gestational age. Nephrogenic zone and cortex at (a) medium and (b) high power. Ureteric bud (UB), ampulla (A), vesicle (V), metanephric mesenchyme (M), and comma-shaped body (C in panel a) do not express CSR. (b) CSR immunostaining in nephrogenic zone is first detected in the upper limb (UL) of S-shaped body, which will develop into the loop of Henle (h), first portion of distal tubule and macula densa (md), and distal convoluted tubule (d). Distal convoluted tubule (DT) and thick ascending limb (TAL) are positive for CSR immunostaining. S-shaped body middle limb (ML) and lower limb (LL), glomeruli (G), collecting duct (CD), proximal tubule (PT), and thick descending limb (TDL) do not express CSR; capsule (C in panel b). Inset in panel a shows ampulla and vesicle with no CSR staining; inset in panel b identifies ampulla and mesenchyme nuclear staining for PAX2. Sections counterstained with hematoxylin. Original magnification: (a) $\times 200$; (b) $\times 400$; insets $\times 600$. Bar $=50 \mu \mathrm{m}$. 


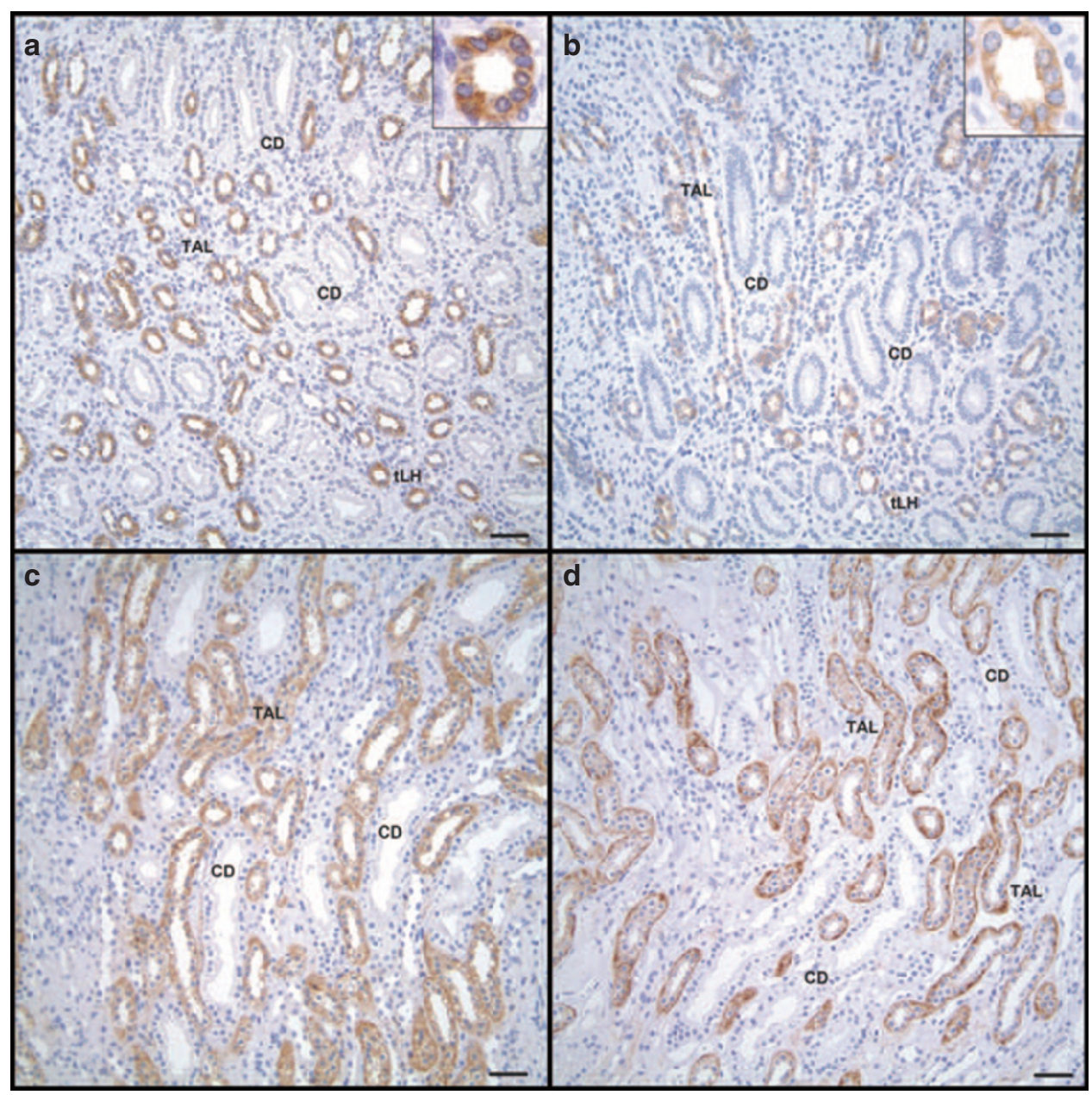

Figure 2. Calcium-sensing receptor (CSR) immunostaining in developing human kidney and mature kidney using (a,c) a monoclonal antibody to CSR and $(\mathbf{b}, \mathbf{d})$ a polyclonal antibody to CSR. (a,b) Kidney, at 21 wk gestational age, at the corticomedullary junction with CSR immunostaining of early thin limb (tLH) and thick ascending limb (TAL) of the loop of Henle, with no staining of collecting duct (CD). (c,d) Adult kidney medulla with immunostaining of CSR in TAL. Staining pattern is basolateral and cytoplasmic (insets in panels $\mathbf{a}$ and $\mathbf{b}$ ). Larger tubules represent collecting ducts which are negative for CSR immunostaining. Sections counterstained with hematoxylin. Original magnification: (a-d) $\times 200$; insets $\times 600$. Bar $=50 \mu \mathrm{m}$.

remaining limited to descending limbs. Developing TAL and DT showed cytoplasmic and basolateral CSR staining of variable intensity with both CSR monoclonal and polyclonal antibodies (Figures 1 and 2).

Mature human kidney tubular segments in the medulla showed immunolocalization of CSR limited to the TAL with both monoclonal and polyclonal antibodies tested (Figure 2c,d). CSR expression was both cytoplasmic and membranous, with overall more intense basolateral staining when compared with the developing kidney. Cortical and medullary CD did not show immunoreactivity for CSR in principal epithelial cells or intercalated cells. Immunoreactivity of nephron segments throughout development and in mature human kidneys did not differ between the CSR monoclonal and polyclonal antibodies used.

\section{Histomorphology and CSR in Developing Cortical Tubular Segments}

Evaluation of CSR immunostaining in cortical and medullary tubular nephron segments was based on the histomorphologic characteristics of tissue sections stained with hematoxylin and eosin and the anatomic location of the tubules (Figure 3 ). Developing cortical tubule segments show differences in morphology as early as $20 \mathrm{wk}$ gestational age. Proximal convoluted tubules (PT) are larger and more numerous compared with the short DT. PT are lined by cuboidal to low columnar cells with abundant eosinophilic granular cytoplasm; centrally located nuclei; and an apical, more eosinophilic brush border (Figure 3a,c). DT show more nuclei per cross section, are lined by cuboidal cells with scant, pale to eosinophilic cytoplasm, apically located nuclei, and absent brush border (Figure 3a,c, inset in panel c). TAL ascends into the cortex and bends over the glomerulus; the tubule segment abutting the glomerular vascular pole becomes the MD, and beyond the MD is the DT. All these distal tubule segments express CSR as seen in Figure 3b,d. PT and glomeruli are negative for CSR.

\section{Dual Immunohistochemistry}

Dual immunohistochemistry studies were performed on four representative developing kidneys (gestational age: 20, 


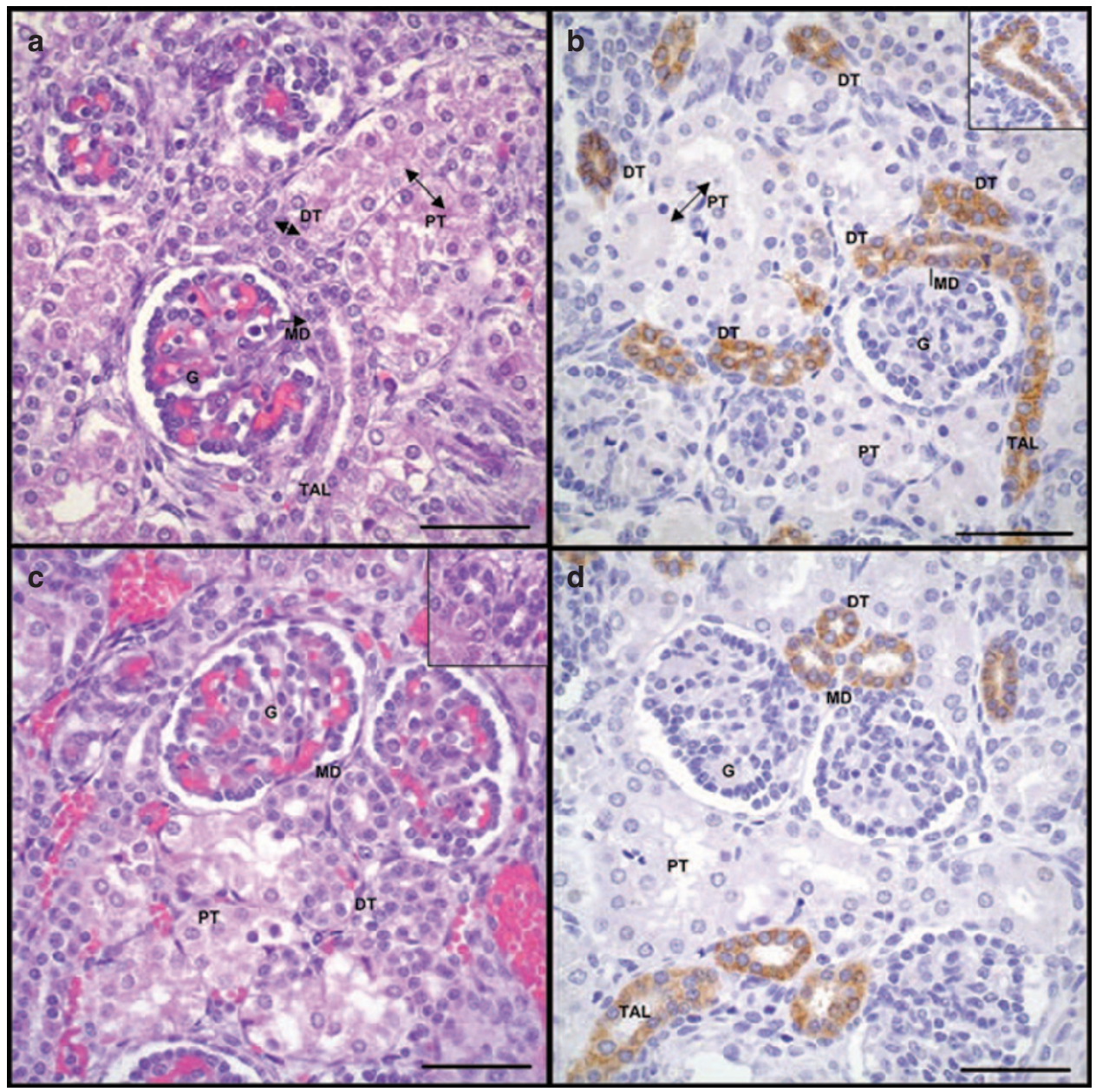

Figure 3. Hematoxylin and eosin (H\&E) and calcium-sensing receptor (CSR) staining of developing human kidney cortex at (a,b) 24 and (c,d) 20 wk gestational age. (a,c) H\&E staining differentiates between cortical proximal and distal tubule segments. Proximal convoluted tubules (PT) are identified by cuboidal to low columnar cells with abundant eosinophilic cytoplasm, centrally located nuclei, and an apical darker staining brush border (double arrow in panel a). The macula densa (MD) is located at the glomerular hilum and represents the end of the thick ascending limb (TAL). Distal convoluted tubules (DT) begin just beyond the MD, are less numerous, and have cuboidal cells with scant cytoplasm, apically located nuclei, and absent brush border. Inset in panel c shows DT tubular cross-sections with darker nuclei (upper right) and PT with abundant cytoplasm and fewer nuclei (lower left). (b,d) CSR immunostaining of TAL, MD at glomerular vascular pole, and DT (inset in panel b). PT and glomeruli (G) are negative for CSR. CSR sections counterstained with hematoxylin. Original magnification: $\times 600$; inset $\times 600$. Bar $=50 \mu \mathrm{m}$.

26,32 , and $37 \mathrm{wk}$ ) and three mature kidneys to substantiate the morphologic interpretation. Dual stains were performed using the CSR monoclonal antibody and, in three mature kidney cases, using the polyclonal antibody.

CSR and CD10. Dual immunohistochemical studies were performed with antibodies to CSR (visualized by brown chromogen) and CD10 (visualized by red chromogen) to further evaluate CSR staining in PT. There was no colocalization of CD10 and CSR in proximal convoluted and straight tubules in developing kidney (Figure 4). CD10-positive glomerular epithelial cells did not express CSR.

CSR and epithelial membrane antigen (EMA). Dual immunohistochemical studies were performed with antibodies to CSR (visualized by brown chromogen) and EMA (visualized by red chromogen) to further evaluate CSR localization in distal nephron segments, including TAL, DT, and cortical and medullary CD. CSR colocalized with EMA-positive TAL and DT in developing and mature human kidney (Figure 5).
Throughout their length, EMA-positive cortical and medullary CD did not stain for CSR.

\section{DISCUSSION}

The data presented here demonstrate that the CSR is present in TAL and DT but is below the level of detection in other nephron segments in developing human kidney. Nephrogenic structures destined to form distal tubule segments are positive for the CSR, but other nephrogenic structures, including ureteric buds, are negative for CSR staining.

In fetal rat kidney, the CSR is expressed in apical epithelial cells of large tubules and branching ureteric buds. In the early postnatal period, there is a dramatic increase in expression of the CSR in rat kidney, principally in the TAL, but also in the CD (23). In mature rat kidney, CSR expression in cortical CD is limited to intercalated cells (24), whereas in inner medullary CD, the CSR is expressed in principal cells (18). In the early stages of human renal development, immature and 


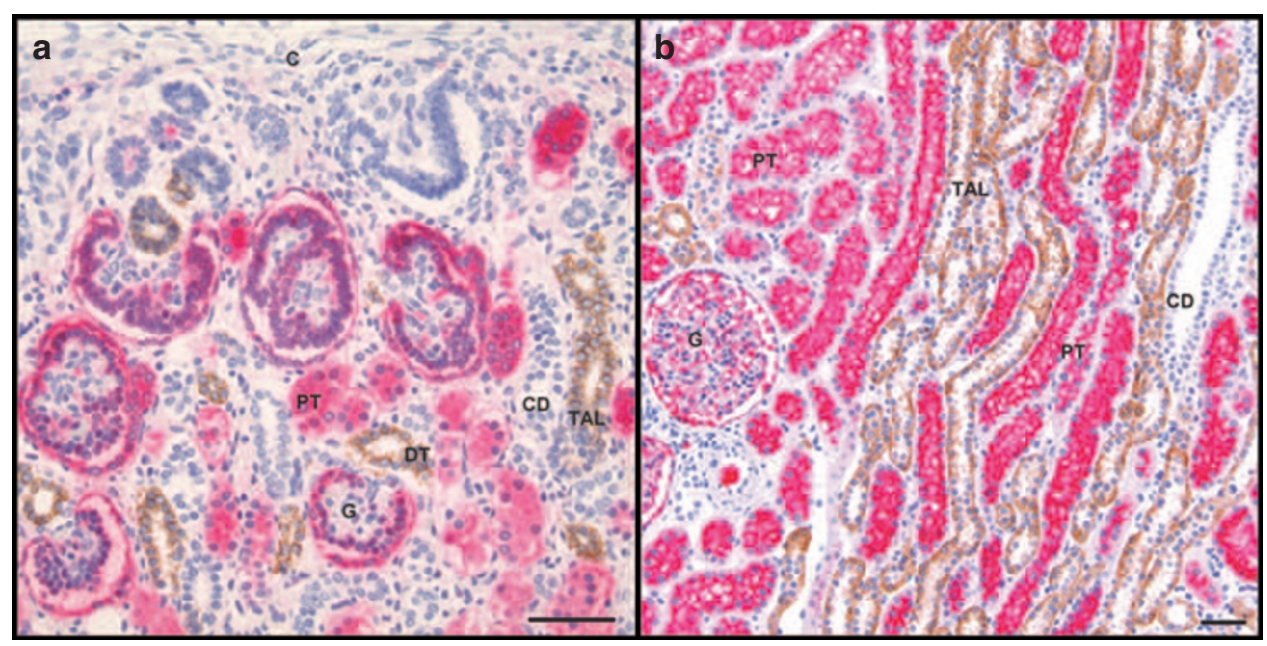

Figure 4. Calcium-sensing receptor (CSR) and CD10 dual immunostaining in (a) developing human kidney at 32 wk gestational age and (b) mature kidney (45 y old). CD10 (red chromogen) identifies glomeruli (G) and proximal tubule (PT), which do not stain with CSR. CSR (brown chromogen) immunostaining of distal tubules (DT) and thick ascending limb (TAL), which do not express CD10. Collecting duct (CD) was negative for CD10 and CSR in both developing and mature human kidneys. Sections counterstained with hematoxylin. Original magnification: (a) $\times 400 ;(\mathbf{b}) \times 200$. Bar $=50 \mu \mathrm{m}$.

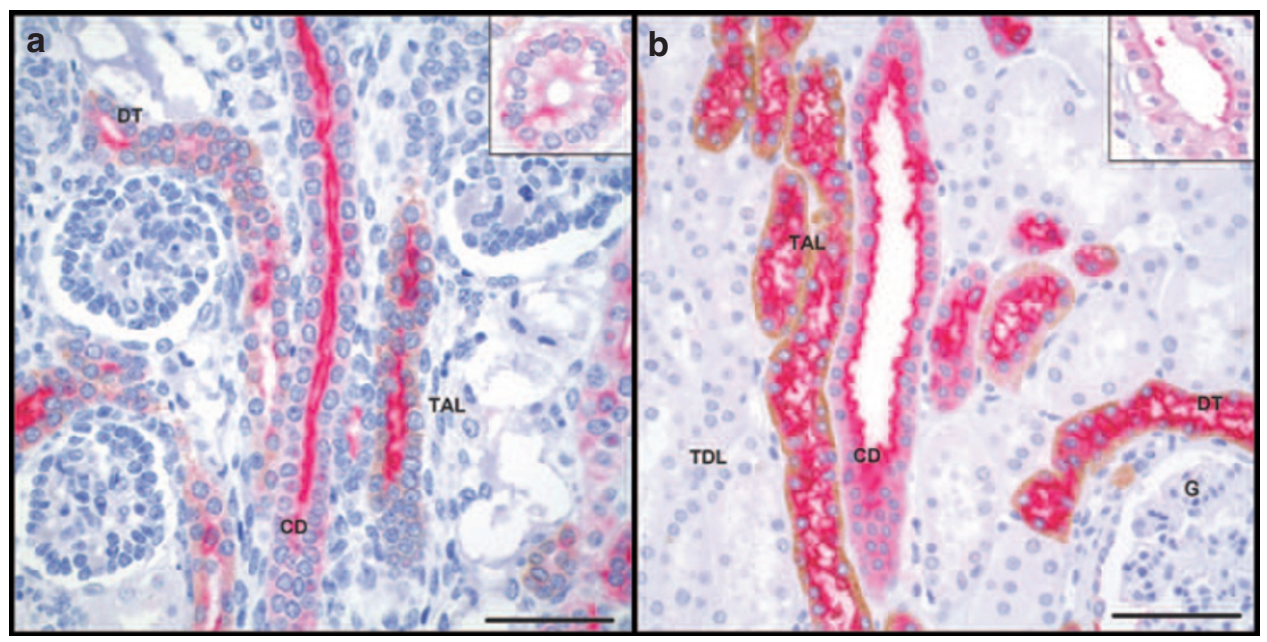

Figure 5. Calcium-sensing receptor (CSR) and epithelial membrane antigen (EMA) dual immunostaining in (a) developing human kidney at 20 wk gestational age and (b) mature kidney (45 y old). EMA (red chromogen) identifies distal nephron segments showing cytoplasmic and apical staining in thick ascending limb (TAL), distal tubule (DT), and collecting duct (CD). CSR (brown chromogen) co-localizes with EMA in DT and TAL, but not in CD (insets), in both developing and mature human kidneys. Sections counterstained with hematoxylin. G, glomeruli. Original magnification: $(\mathbf{a}, \mathbf{b}) \times 600$; insets $\times 1,000$. Bar $=50 \mu \mathrm{m}$.

undifferentiated intercalated cells represent $3-4 \%$ of epithelial $\mathrm{CD}$ cells and are identified in the inner medulla $\mathrm{CD}$, whereas later in gestation and postnatally, intercalated cells are present in outer medullary and cortical CD and account for 5-10\% of CD cells (25). We did not identify CSR localization throughout the $\mathrm{CD}$ during human renal development, nor did we identify CSR in other Wolffian duct-derived structures such as ureteric buds, which are positive for PAX2. A role for the CD CSR in preventing calcium stone disease, via enhanced free water excretion in response to increased urinary calcium, has been proposed (17), but physiologic studies by Bergsland (21) and Lam (20) failed to demonstrate a change in urine volume induced by increased urinary calcium, observations that challenge the concept that the urinary calcium plays a role in regulation of urine volume. Because we did not observe staining for the CSR in developing $\mathrm{CD}$, it is possible that absent or diminished CSR function in this developing nephron segment is a factor in the pathogenesis of nephrocalcinosis in these infants. Two studies have addressed localization of the CSR in mature human kidney. Using rabbit anti-CSR antiserum against bovine CSR (amino acids 215-237), Sands et al. demonstrated CSR staining of CD in frozen sections of human kidney (18). In contrast, Loupe recently found that CSR staining of human kidney is limited to the TAL, using a mouse monoclonal antibody to amino acids $214-235$ of the human CSR, as well as a polyclonal antibody directed against the C-terminal tail of the rat CSR (26). We observed positive staining for the CSR in cells of the TAL, but not in cells of adjacent proximal tubule segments, and $\mathrm{CD}$. This distinct pattern of CSR staining, in which CSR-positive tubules could be clearly distinguished from CSR-negative tubules, was noted as early as $20 \mathrm{wk}$ of development, when active formation of nephrons 


\section{Articles | Crisietal.}

takes place in the subcapsular nephrogenic zone. CSR immunolocalization was observed in the same nephrogenic zone structures at 20 to 32 wk gestational age. Our observations suggest that functional differentiation of tubular cells is occurring at this stage in development. In the nephrogenic mesenchyme, we observed CSR immunolocalization in the upper limb of the S-shaped body, which is destined to develop into distal structures, including TAL and DT. Approximately $14 \%$ of S-shaped bodies at the stage of hemispheric cavity formation and approximately $80 \%$ of late S-shaped bodies demonstrated CSR staining in upper limb structures. Taken together, these observations suggest that CSR expression is a developmental phenomenon, occurring as these cells differentiate into their respective nephron components as nephrogenesis progresses.

PT did not stain for the CSR in developing human kidney. It has been suggested that aminoglycoside toxicity is mediated by the CSR via proximal tubular cell apoptosis (27). Because many premature infants receive aminoglycosides as treatment for presumed sepsis, it is reassuring to note that we did not identify CSR expression in PT, which may explain the observation that acute aminoglycoside nephrotoxicity is an uncommon event in premature infants. In animal models, however, PT has been shown to express CSR (24).

In our immunohistochemical studies of developing human kidney, the CSR was below the level of detection in PT and CD. However, immunohistologic studies have identified the CSR in these nephron segments in human as well as animal kidney, and physiologic studies of cells from these regions document functions attributable to this receptor. There are a number of reasons that may explain the lack of immunolocalization of CSR in these developing nephron segments. At this stage in human development, the CSR may not be expressed in immature or undifferentiated tubular cells, including intercalated cells, in these developing nephron segments. Alternatively, the CSR may be present in developing PT and CD at a very low density on the cell membrane when compared with cells of the TAL, but still capable of physiologic response. The membrane density of this receptor may increase as a normal developmental sequence or in response to changes in peritubular or luminal calcium concentration. In the work presented, using both monoclonal and polyclonal CSR antibodies, CSR protein expression in developing human $\mathrm{CD}$ and $\mathrm{PT}$ is either absent or below the detection limits of immunohistochemistry with the antibodies used in this study. It is possible that regardless of antigen retrieval, in formalin-fixed tissue, the targeted epitopes of the antibodies used may not be available in PT or CD for antigen-antibody interaction and therefore not detected by immunohistochemical methods. To obtain answers to these questions, studies of developing human $\mathrm{CD}$ and PT using more sensitive techniques are required. We could not confidently utilize in situ hybridization in our study of archival formalin-fixed, paraffin-embedded renal tissue because of RNA degradation.

The role of the renal CSR during fetal development has been studied. Mice targeted for deletion of the CSR die soon after birth because of severe hyperparathyroidism and hypercalcemia (28), whereas mice deficient in both the CSR and parathyroid hormone develop normally, suggesting that the deleterious effects of CSR gene deletion on the fetus are caused by hyperparathyroidism and hypercalcemia (29). Kovacs et al. found that amniotic fluid calcium concentration in mice lacking the CSR gene is elevated in comparison to normal mice, reflecting the relative hyperparathyroidism or hypercalcemia. Furthermore, these authors found that amniotic fluid calcium was similar in mice heterozygous and homozygous for deletion of the CSR, and speculated that fetal tubular handling of calcium is not affected by CSR gene disruption (30).

The CSR may play a role in preventing calcium-related disorders in premature infants. In the newborn period, hypercalcemia is uncommon, occurring in association with idiopathic infantile hypercalcemia and primary or secondary hyperparathyroidism; iatrogenic hypercalcemia may be the most common cause (31). Loss-of-function mutations of the CSR gene are associated with hypocalciuria and hypercalcemia. Given that hypercalcemia is rare in the newborn period, it is likely that the CSR, expressed in the TAL, participates in calcium homeostasis during early development by increasing urinary calcium excretion in response to elevated serum calcium. Nephrocalcinosis has been reported to occur in about $15 \%$ of premature infants and may also occur in association with gain-of-function mutations of the CSR, which are characterized by hypocalcemia and hypercalciuria. It is possible to speculate that impaired regulation of CSR function in the TAL, leading to an exaggerated response to serum calcium and increased urinary calcium excretion, could play a role in the development of nephrocalcinosis in premature infants. Because the CSR is not expressed in the CD at any point in development, and because recent data fail to demonstrate a role for calcium in osmoregulation, it is unlikely that the CSR plays a role in nephrocalcinosis via dilution of urine in response to increased calcium.

In summary, the CSR is present in the developing human kidney in TAL and DT, as well as in early nephrogenic structures destined to form these nephron segments. The CSR could not be detected in developing proximal tubule segments and $\mathrm{CD}$, nor in nephrogenic structures destined to form these nephron segments. Presence of the CSR is characteristic of developing human TAL, where this receptor may play a role in renal calcium regulation in premature infants.

\section{METHODS}

This study was based on archival autopsy material and was approved by the institutional review board of Baystate Medical Center. The renal tissue studied was obtained after death as a result of intrauterine spontaneous fetal demise, or from infants who died as a result of the complications of prematurity. A search of the pathology CoPath electronic files was performed for autopsies between 20 and $40 \mathrm{wk}$ of intrauterine development. All tissues were archival formalin-fixed and paraffin-embedded tissue blocks. Hematoxylin and eosin-stained kidney tissue sections were reviewed to evaluate for adequacy of tissue preservation and for presence of an intact renal capsule. Exclusion criteria included genetic abnormalities (based on cytogenetic testing or morphology) and inadequate preservation of renal histomorphology. Mature non-neoplastic renal tissue was obtained from five tumor nephrectomies. 


\section{Immunohistochemistry}

Immunohistochemical staining was performed on a DakoCytomation AutoStainer using the Dako ADVANCE HRP/DAB staining kit protocol in accordance with the Signet Acuity data sheet (Dako, Carpenteria, CA), as previously reported (32). The antibodies used included mouse monoclonal antibody anti-CSR clone 5C10 corresponding to amino acids $214-235$ of the human CSR (1:10,000; Novus Biologicals, Littleton, CO) (33), rabbit polyclonal antibody anti-CSR corresponding to amino acids 345-359 of the bovine CSR (1:500, generous gift of Edward M. Brown) (34), monoclonal antihuman EMA clone E29 (1:4,000; Dako) (35), monoclonal CD10 clone 56C6 (1:200; Vector Laboratories, Burlingame, CA) (36), and polyclonal antibody to PAX2 (1:100; Invitrogen, Camarillo, CA) (37). Antigen retrieval for the CSR antibodies and CD10 was performed with EDTA pH9.0; antigen retrieval for EMA and PAX2 was performed in a microwave using a $1 \times$ working concentration of BioGenex (Fremont, CA) Concentrated $(10 \times)$ Sodium Citrate Antigen Retrieval Solution Plus. Single immunohistochemical stains were visualized with diaminobenzidine chromogen (DAB, brown). Dual immunohistochemical staining was performed using a dual reaction kit. The first reaction included the CSR monoclonal or polyclonal antibody (DAB, brown); the second reaction included antibodies to EMA or CD10 (permanent red). Positive and negative controls omitting the primary antibody, and an IgG polyclonal isotype control (PA1-27414, Pierce BioTechnology, Rockford, IL), were run in parallel.

\section{Histomorphology and Evaluation of CSR Immunoreactivity in Nephron Structures}

Microscopic evaluation of CSR immunostaining was performed by evaluating the entire kidney tissue at medium and high power. In the subcapsular nephrogenic zone, structures that were evaluated included ureteric buds, vesicles, comma-shaped bodies, S-shaped bodies (upper, middle, and lower limbs), and primitive glomeruli. CSR staining in the nephrogenic zone was evaluated by calculating the percentage of CSR-positive structures after the total number of identifiable nephrogenic structures was counted.

Evaluation of CSR immunostaining in cortical and medullary tubular nephron segments was based on the histomorphologic characteristics on hematoxylin and eosin-stained tissue sections (32), association with specific antigens (33-37), and location within the renal parenchyma.

\section{ACKNOWLEDGMENT}

The authors thank Brooke Bentley for technical assistance.

\section{STATEMENT OF FINANCIAL SUPPORT}

This study was supported by Baystate Health Systems Incubator Grant SP07-068, Baystate Medical Center, Springfield, MA (Crisi); and Tsacoyeanes Pediatric Renal Research Award, National Kidney Foundation serving New England, Norwood, MA (Campfield).

Disclosure: The authors declare no conflict of interest.

\section{REFERENCES}

1. Rauch F, Schoenau E. Skeletal development in premature infants: a review of bone physiology beyond nutritional aspects. Arch Dis Child Fetal Neonatal Ed 2002;86:F82-5.

2. Hufnagle KG, Khan SN, Penn D, Cacciarelli A, Williams P. Renal calcifications: a complication of long-term furosemide therapy in preterm infants. Pediatrics 1982;70:360-3.

3. Jacinto JS, Modanlou HD, Crade M, Strauss AA, Bosu SK. Renal calcification incidence in very low birth weight infants. Pediatrics 1988;81: 31-5.

4. Downing GJ, Egelhoff JC, Daily DK, Thomas MK, Alon U. Kidney function in very low birth weight infants with furosemide-related renal calcifications at ages 1 to 2 years. J Pediatr 1992;120(4 Pt 1):599-604.

5. Ezzedeen F, Adelman RD, Ahlfors CE. Renal calcification in preterm infants: pathophysiology and long-term sequelae. J Pediatr 1988;113: $532-9$.
6. Kist-van Holthe JE, van Zwieten PH, Schell-Feith EA, et al. Is nephrocalcinosis in preterm neonates harmful for long-term blood pressure and renal function? Pediatrics 2007;119:468-75.

7. Narendra A, White MP, Rolton HA, et al. Nephrocalcinosis in preterm babies. Arch Dis Child Fetal Neonatal Ed 2001;85:F207-13.

8. Gimpel C, Krause A, Franck P, Krueger M, von Schnakenburg C. Exposure to furosemide as the strongest risk factor for nephrocalcinosis in preterm infants. Pediatr Int 2010;52:51-6.

9. Campfield T, Braden G, Flynn-Valone P, Powell S. Effect of diuretics on urinary oxalate, calcium, and sodium excretion in very low birth weight infants. Pediatrics 1997;99:814-8.

10. Schell-Feith EA, Kist-van Holthe JE, Conneman N, et al. Etiology of nephrocalcinosis in preterm neonates: association of nutritional intake and urinary parameters. Kidney Int 2000;58:2102-10.

11. Vikse BE, Irgens LM, Leivestad T, Hallan S, Iversen BM. Low birth weight increases risk for end-stage renal disease. J Am Soc Nephrol 2008;19: $151-7$.

12. White SL, Perkovic V, Cass A, et al. Is low birth weight an antecedent of CKD in later life? A systematic review of observational studies. Am J Kidney Dis 2009;54:248-61.

13. Riccardi D, Brown EM. Physiology and pathophysiology of the calciumsensing receptor in the kidney. Am J Physiol Renal Physiol 2010;298: F485-99.

14. Di Stefano A, Wittner M, Nitschke R, et al. Effects of parathyroid hormone and calcitonin on $\mathrm{Na}+, \mathrm{Cl}-, \mathrm{K}+, \mathrm{Mg} 2+$ and $\mathrm{Ca} 2+$ transport in corti$\mathrm{cal}$ and medullary thick ascending limbs of mouse kidney. Pflugers Arch 1990;417:161-7.

15. Hebert SC, Brown EM, Harris HW. Role of the $\mathrm{Ca}(2+)$-sensing receptor in divalent mineral ion homeostasis. J Exp Biol 1997;200(Pt 2): 295-302.

16. Desfleurs E, Wittner M, Simeone S, et al. Calcium-sensing receptor: regulation of electrolyte transport in the thick ascending limb of Henle's loop. Kidney Blood Press Res 1998;21:401-12.

17. Varghese J, Rich T, Jimenez C. Benign familial hypocalciuric hypercalcemia. Endocr Pract 2010;6:1-16.

18. Sands JM, Naruse M, Baum M, et al. Apical extracellular calcium/polyvalent cation-sensing receptor regulates vasopressin-elicited water permeability in rat kidney inner medullary collecting duct. J Clin Invest 1997;99:1399-405.

19. Procino G, Mastrofrancesco L, Mira A, et al. Aquaporin 2 and apical calcium-sensing receptor: new players in polyuric disorders associated with hypercalciuria. Semin Nephrol 2008;28:297-305.

20. Lam GS, Asplin JR, Halperin ML. Does a high concentration of calcium in the urine cause an important renal concentrating defect in human subjects? Clin Sci 2000;98:313-9.

21. Bergsland KJ, Coe FL, Gillen DL, Worcester EM. A test of the hypothesis that the collecting duct calcium-sensing receptor limits rise of urine calcium molarity in hypercalciuric calcium kidney stone formers. Am J Physiol Renal Physiol 2009;297:F1017-23.

22. Renkema KY, Bindels RJ, Hoenderop JG. Role of the calcium-sensing receptor in reducing the risk for calcium stones. Clin J Am Soc Nephrol 2011;6:2076-82.

23. Chattopadhyay N, Baum M, Bai M, et al. Ontogeny of the extracellular calcium-sensing receptor in rat kidney. Am J Physiol 1996;271(3 Pt 2): F736-43.

24. Riccardi D, Hall AE, Chattopadhyay N, Xu JZ, Brown EM, Hebert SC. Localization of the extracellular Ca2+/polyvalent cation-sensing protein in rat kidney. Am J Physiol 1998;274(3 Pt 2):F611-22.

25. Hiatt MJ, Ivanova L, Toran N, Tarantal AF, Matsell DG. Remodeling of the fetal collecting duct epithelium. Am J Pathol 2010;176:630-7.

26. Loupy A, Ramakrishnan SK, Wootla B, et al. PTH-independent regulation of blood calcium concentration by the calcium-sensing receptor. J Clin Invest 2012;122:3355-67.

27. Ward DT, Maldonado-Pérez D, Hollins L, Riccardi D. Aminoglycosides induce acute cell signaling and chronic cell death in renal cells that express the calcium-sensing receptor. J Am Soc Nephrol 2005;16: $1236-44$. 


\section{Articles $\mid$ Crisiet al.}

28. Ho C, Conner DA, Pollak MR, et al. A mouse model of human familial hypocalciuric hypercalcemia and neonatal severe hyperparathyroidism. Nat Genet 1995;11:389-94.

29. Kos CH, Karaplis AC, Peng JB, et al. The calcium-sensing receptor is required for normal calcium homeostasis independent of parathyroid hormone. J Clin Invest 2003;111:1021-8.

30. Kovacs CS, Ho-Pao CL, Hunzelman JL, et al. Regulation of murine fetalplacental calcium metabolism by the calcium-sensing receptor. J Clin Invest 1998;101:2812-20.

31. Rodd C, Goodyer P. Hypercalcemia of the newborn: etiology, evaluation, and management. Pediatr Nephrol 1999;13:542-7.

32. Crisi GM, Marconi SA, Rockwell GF, Braden GL, Campfield TJ. Immunolocalization of CD44 and osteopontin in developing human kidney. Pediatr Res 2009;65:79-84.

33. Goldsmith PK, Fan G, Miller JL, Rogers KV, Spiegel AM. Monoclonal antibodies against synthetic peptides corresponding to the extracellular domain of the human $\mathrm{Ca} 2+$ receptor: characterization and use in studying concanavalin A inhibition. J Bone Miner Res 1997;12: 1780-8.

34. Kifor O, Moore FD Jr, Wang $\mathrm{P}$, et al. Reduced immunostaining for the extracellular $\mathrm{Ca} 2+$-sensing receptor in primary and uremic secondary hyperparathyroidism. J Clin Endocrinol Metab 1996;81: 1598-606.

35. Fleming S, Lindop GB, Gibson AA. The distribution of epithelial membrane antigen in the kidney and its tumours. Histopathology 1985;9: 729-39.

36. Holm-Nielsen P, Pallesen G. Expression of segment-specific antigens in the human nephron and in renal epithelial tumors. APMIS Suppl 1988;4: 48-55.

37. Eccles MR, He S, Legge M, et al. PAX genes in development and disease: the role of PAX2 in urogenital tract development. Int J Dev Biol 2002;46: 535-44. 